\title{
Prospective Randomized Controlled Clinical Study of Physiological Ventricular Rhythm Resetting in Patients with Atrial Fibrillation and Heart Failure: Rationale and Design
}

\section{Zhixin JIANG}

Jiangsu Province People's Hospital and Nanjing Medical University First Affiliated Hospital: Jiangsu Province Hospital and Nanjing Medical University First Affiliated Hospital

\section{Wen YANG}

Jiangsu Province People's Hospital and Nanjing Medical University First Affiliated Hospital: Jiangsu Province Hospital and Nanjing Medical University First Affiliated Hospital

\section{Tian WU}

Jiangsu Province People's Hospital and Nanjing Medical University First Affiliated Hospital: Jiangsu

Province Hospital and Nanjing Medical University First Affiliated Hospital

\section{Yiting LV}

Jiangsu Province People's Hospital and Nanjing Medical University First Affiliated Hospital: Jiangsu

Province Hospital and Nanjing Medical University First Affiliated Hospital

Jie GENG

Jiangsu Province People's Hospital and Nanjing Medical University First Affiliated Hospital: Jiangsu

Province Hospital and Nanjing Medical University First Affiliated Hospital

Jingwen HU

Jiangsu Province People's Hospital and Nanjing Medical University First Affiliated Hospital: Jiangsu Province Hospital and Nanjing Medical University First Affiliated Hospital

\section{Xiujuan ZHOU}

Jiangsu Province People's Hospital and Nanjing Medical University First Affiliated Hospital: Jiangsu Province Hospital and Nanjing Medical University First Affiliated Hospital

qijun Shan ( $\nabla$ qjshan@njmu.edu.cn )

Jiangsu Province People's Hospital and Nanjing Medical University First Affiliated Hospital: Jiangsu Province Hospital and Nanjing Medical University First Affiliated Hospital https://orcid.org/0000-00018783-8057

\section{Research Article}

Keywords: atrial fibrillation, heart failure, physiological ventricular rhythm resetting, medical therapy 
Posted Date: February 2nd, 2022

DOI: https://doi.org/10.21203/rs.3.rs-1218339/v1

License: (c) (i) This work is licensed under a Creative Commons Attribution 4.0 International License. Read Full License 


\section{Abstract \\ Background}

Atrial fibrillation (AF) and heart failure (HF) frequently co-exist. HF may present in $20-30 \%$ of AF patients. Both $\mathrm{AF}$ and $\mathrm{HF}$ facilitate the occurrence and aggravate the prognosis of each other, which significantly affects the quality of life and increases mortality. This paper describes the rationale and the design of the trial 'Prospective randomized controlled clinical study of physiological ventricular rhythm resetting in patients with atrial fibrillation and heart failure' and the need to improve the treatment of the patients with $\mathrm{AF}$ and $\mathrm{HF}$.

\section{Methods}

This study is a registry-based, prospective, randomized, parallel, single-center trial. The primary aim is to determine whether the physiological ventricular rhythm resetting (atrioventricular node ablation with physiological LBBP) can improve clinical outcomes (all-cause mortality or rehospitalization for HF) compared with medical rate control. A total of 110 patients will be enrolled in the trial and followed up for 1 year.

\section{Summary}

The cardioversion of $\mathrm{AF}$ is a major treatment for $\mathrm{AF}$ patients with HF. However, the recurrence rate of atrial fibrillation ablation is high and part of these patients are not suitable for ablation, which raised the difficulty to treat such patients. Some observational studies found that AVNA with physiological pacing could improve the prognosis of these patients. Thus, results from this trial may influence future treatment strategies and guidelines specific to AF patients with HF.

\section{Background And Rationale}

Atrial fibrillation (AF) and heart failure (HF) frequently co-exist. HF may present in $20-30 \%$ of $\mathrm{AF}$ patients ${ }^{[1]}$. Both $\mathrm{AF}$ and $\mathrm{HF}$ facilitate the occurrence and aggravate the prognosis of each other, which significantly affects the quality of life and increases mortality. The management of AF patients with $\mathrm{HF}$ is extremely challenging.

Rate-control and rhythm-control of AF are central to the treatment of these patients ${ }^{[1]}$. The optimal heart rate in patients with $\mathrm{AF}$ remains unclear. Compared with strict rate-control (rest heart rate $\$ 80 \mathrm{bpm}$,moderate activity heart rate $\$ 110 \mathrm{bpm}$ ), recent studies found that lenient rate-control (rest heart rate $₫ 110 \mathrm{bpm}$ ) showed no significant difference in composite clinical endpoints, NYHA class and

hospitalization ${ }^{[2-5]}$. Rhythm control includes two ways, drugs and catheter ablation. A meta-analysis found that rhythm control by drugs had no advantage over rate control. Rhythm control by catheter 
ablation could reduce all-cause mortality and rehospitalization for $\mathrm{HF}$, improve quality of life, and increase LVEF by $6.8 \%$ compared with medical therapy ${ }^{[6]}$. However, due to the high recurrence rate and surgical cost of AF ablation, whether catheter ablation for these patients remains a difficult decision ${ }^{[6-8]}$.

Atrioventricular node ablation (AVNA) and pacing therapy (Ablation and Pacing Therapy, APT) have proved to improve the quality of life and increase LVEF as catheter ablation ${ }^{[9]}$. However, APT needs longterm right ventricular apical pacing (RVAP), which may further aggravate HF by leading to ventricular electromechanical dyssynchrony ${ }^{[10]}$. Hence, APT was only served as the last strategy for patients who are unresponsive or intolerant to intensive rate and rhythm control therapy ${ }^{[1]}$. According to recent studies, patients with AF and HF can benefit from physiological APT strategy [AVNA with His bundle pacing (HBP) or left bundle branch pacing (LBBP)] even the heart rate controlled very well ${ }^{[11,12]}$. This suggests that irregular ventricular rhythm may be an important reversible factor in patients with AF and HF.

Here, we describe a new concept of physiological ventricular rhythm resetting (AVNA with physiological pacing) and designed a prospective, randomized clinical trial to determine whether the physiological LBBP ventricular rhythm resetting (AVNA with LBBP) can improve clinical outcomes (all-cause mortality or rehospitalization for HF) compared with medical rate-control treatment.

\section{Methods}

The physiological ventricular rhythm resetting is a registry-based, prospective, randomized, parallel, single-center trial. The primary aim is to determine whether the irregular ventricular rhythm is an important reversible factor for patients with $\mathrm{AF}$ and $\mathrm{HF}$ and whether physiological ventricular resetting reduces the composite clinical endpoints, including the rate of all-cause mortality and rehospitalization for HF. The second aims are to determine whether physiological ventricular resetting improves cardiac function, ventricular remodeling, and the quality of life. The study was approved by Ethics Review Boards in China. No. of the ethic committee: 2021-SRFA-417. Clinical Trials Registration number: ChiCTR2100047640.

The authors are solely responsible for the design and conduct of this study, all study analyses, the drafting and editing of the paper, and its final contents.

\section{Study Population And Follow Up}

Inclusion and exclusion criteria in Table I. The study concerned with patients enrolled in the first affiliated hospital of Nanjing Medical University from July 2021 to June 2023. 110 eligible patients are expected to be included in the trial with a 1:1 allocation to receive treatment with either physiological ventricular resetting group or medical rate control group (Figure I) with 1 year follow-up. A computer-generated list of random treatment allocations will be used to randomize enrolled subjects. If a subject is randomized to the physiological ventricular resetting group, the procedure should implement within 30 days. 
All subjects should undergo a detailed evaluation before the randomization, including baseline echocardiography (TTE), 24h-Holter electrographic monitoring, pro-B-type natriuretic peptide (pro-BNP), NYHA class, 6-minute walk test (6MWT), and the six-item specific symptom scale score (SSS). All subjects will be followed up at 1 week, 1, 3, 6, and 12 months after discharge. If a subject experienced increased edema, decreased urine, or chest tightness during follow-up, pro-BNP and TTE tests should be evaluated as early as possible. Adverse events will be recorded and handled promptly.

\section{Procedure}

All patients will receive standard treatment for AF and HF. $\beta$-blocker is the first choice for rate control (digoxin can be used when $\beta$-blocker is contraindications or not effective), aiming to make heart rate below 110 beats per minute. Any changes in the medication regimen should be written and documented.

The patients of the physiological ventricular resetting group will undergo extra AVNA and LBBP. Before the procedure, patients need to sign the consent form.

LBBP: The procedure is performed under local anesthesia. The 7-Fr guiding catheter (Model C315-S10; Medtronic Inc) and the pacing electrodes (models 3830, Medtronic Inc)were advanced into the right ventricular transvenous by left subclavian or axillary vein puncture. Move the catheter towards the apex of the heart by $1.5-2.0 \mathrm{~cm}$ after mapping His bundle potential. Then the pacing lead was perpendicularly screwed through the interventricular septum (IVS) to the LV septal sub-endocardium of the LBB region. The paced QRS morphology and duration, pacing impedance were assessed and recorded frequently via unipolar pacing. Intra-catheter angiography was performed at the left anterior oblique position of $40^{\circ}$ to assess the position of the electrodes and the presence of perforation. The pacing QRS and the peak time of the left ventricle were measured after high-voltage and low-voltage single-pole pacing. A pacing threshold below1.5V@0.5ms is acceptable. During the procedure, if the left interventricular septal surface is penetrated during the screwing of the electrode, the electrode should be withdrawn, replaced, and reimplanted. All pacemakers use the VVI model and determine if defibrillation function is required according to the guidelines. The lower rate of pacemaker sets according to the mean heart rate of 24hHolter, to ensure the heart rate is at the same level before and after the procedure. The lower rate of the pacemaker will be set according to the doctor's request and the patient's condition after the trial.

AVNA: We will perform AVN ablation after successful LBBP at the same procedure time. AVNA was performed using the quadripolar 7-Fr 4-mm tip ablation catheter. An 8.0F venous sheath is inserted through the right femoral vein. The key point is to record his potential after sent the ablation catheter into the right atrium. The methods for AVNA are described as follows: (i) The compact AV node was ablated from the atrial side marked by HIS potential. (ii) The ablation catheter was positioned at least $10 \mathrm{~mm}$ away from the LBBP electrode tip. (iii) The ablation power was set at $30-50 \mathrm{~W}$ and temperature at $43^{\circ} \mathrm{C}$ for a duration of 60-120s. The criteria of successful ablations are as follows: 1) persistent complete atrioventricular block; 2) the left bundle capture threshold and QRS morphology remain unchanged after ablation. 


\section{Clinical Endpoints}

The primary endpoints are all-cause mortality and rehospitalization for HF. All-cause mortality is defined as the percentage of deaths for any cause. Rehospitalization for $\mathrm{HF}$ is defined as requiring hospitalization due to worsening symptoms of HF or AF.

The secondary endpoints are the changes in cardiac structure and function. Left atrial diameter (LAD), left ventricular end-diastolic diameter (LVEDD), left ventricular end-systolic diameter (LVESD), and left ventricular ejection fraction (LVEF) will be analyzed by TTE. Pro-BNP will also be examined to evaluate cardiac function. The functional status of general health-related quality of life will be measured by changes in the classification of NYHA, the distance of 6MWT, and the scores of the SSS scale (Table II).

\section{Safety}

Safety endpoints will be collected at visit or telephone interviews at 1, 3, 6, and 12 months post the procedure. Both the intraoperative and long-term adverse events will be well documented. Adverse events define as pneumothorax, hemopneumothorax, hematoma, pericardial effusion, an episode of acute HF, deteriorating quality of life, rehospitalization for HF and sudden death. Among them, a prolonged hospitalization, disability, impair work capacity or endanger life or death are defined as severe adverse events. The trials will be suspended if more than one subject experience severe adverse events or over half of the subjects experience adverse events. Request the evaluation of relevant experts before deciding whether to continue the trial.

\section{Statistics Methods}

The study will be considered a success if the primary endpoint is met. The expected rate of success is estimated to be an increased LVEF of $7.4 \%(S D=11.7)$, based on the data from historical trials ${ }^{[6,11]}$. Given a one-side test significance level of $2.5 \%$, a total of 88 randomized subjects will provide $90 \%$ power to demonstrate superiority after adjusting for attrition. Consider the rate of loss to follow up maybe $20 \%$, 110 subjects will be required.

Continuous variables will be expressed as mean \pm standard deviation and categorical variables will be expressed as percentages. Paired comparisons were made using a Student's t-test for continuous variables. The Chi-squared or Fisher's test will be used for categorical data. Cox Proportional Risk regression model will be used to assess the risk ratio $(H R)$ for different treatment regimens. P-value $\leq$ 0.05 was considered significant. Data management and analysis will be applied with SPSS v20.0 (IBM Corp).

\section{Results}

The study began enrolling patients in July 2021. As of November 2021, our center has randomized six patients. The majority were women (83.33\%) and the mean age was 65(SD 9.3) years. 


\section{Discussion}

Although the treatment for AF and HF has made great progress, it is still extremely difficult to deal with, which seriously affects the quality of life and increases mortality. In current guidelines, APT is only used as the ultimate option for patients who are unresponsive or intolerant to intensive rate and rhythm control therapy ${ }^{[1]}$. We analyzed the advantages, and disadvantages of different forms of APT ${ }^{[9-11,13-16]}$ (Table III). Studies found that regular RVAP can improve immediate hemodynamics compared with irregular RVAP of the same heart rate ${ }^{[9]}$. Nevertheless, the long-term clinical efficacy of AVNA with RVAP did not decrease rehospitalization for HF and mortality. The reason could be the mode of pacing, because RVAP may lead to ventricular mechanical dyssynchrony, and the long-term and high percentage of RVAP could induce cardiomyopathy ${ }^{[10]}$. To overcome the problem, biventricular pacing began to be used in APT. APAF-CRT study compared the efficacy of AVNA with biventricular pacing therapy and medical therapy. The results showed that AVNA with biventricular pacing therapy was superior to medical therapy in reducing the incidence of HF, shortening hospitalization, improving the quality of life, and reducing allcause mortality ${ }^{[13,14]}$. This study proved the efficacy of biventricular pacing but we should not ignore its disadvantages, including high incidence of nonresponse, high operative difficulty, and high cost ${ }^{[15-17]}$. With the emerge of physiological pacing, Wu found that patients with AF and HF could benefit from a new type of APT, AVNA with HBP or LBBP [11]. They proved that AVNA with HBP or LBBP could both improve cardiac function even in the patients with strict rate-control.

Based on the studies above, we speculate that irregular ventricular rhythm is an important reversible factor in patients with AF and HF. Both regular ventricular rhythm and physiological pacing are indispensable for the treatment of the disease.

We propose a new concept of physiological ventricular rhythm resetting. It is a theoretical innovation and is different from the rhythm-control or rate-control strategies for AF. Physiological ventricular rhythm resetting is a combination of both and is expected to become a major treatment strategy for patients with AF and HF. However, there are no large-scale randomized controlled trials to prove the safety and efficacy of this treatment.

Compared with HBP, LBBP has a higher success rate, and the pacing lead is more stable and secure, which can benefit more patients. Hence, we choose LBBP as the physiological pacing strategy. Based on this, we design a prospective, randomized clinical trial to determine whether the physiological ventricular rhythm resetting (AVNA with LBBP) can improve clinical outcomes (all-cause mortality or rehospitalization for HF) compared with medical rate control. The results may eventually improve the existing APT guideline evidence level.

\section{Declarations}

\section{Funding}


The authors declare that no funds, grants, or other support were received during the preparation of this manuscript.

\section{Competing Interests}

The authors have no relevant financial or non-financial interests to disclose.

\section{Author Contributions}

All authors intellectually contributed to the study protocol development including study design, trial intervention, inclusion and exclusion criteria of patients, measurement of variable and endpoints, and statistical analysis.

\section{Ethics approval}

The study was approved by Ethics Review Boards in China. No. of the ethic committee: 2021-SRFA-417. Clinical Trials Registration number: ChiCTR2100047640.

\section{Consent to participate}

Informed consent must be obtained from all individual participants included in the study.

\section{Consent to publish}

The authors affirm that human research participants provided informed consent for publication.

\section{Data Availability Statements}

The data that support the findings of this study are available from the corresponding author upon reasonable request.

\section{References}

1. Hindricks G, Potpara T, Dagres N, et al.2020 ESC Guidelines for the diagnosis and management of atrial fibrillation developed in collaboration with the European Association of Cardio-Thoracic Surgery (EACTS) $₫ T$ The Task Force for the diagnosis and management of atrial fibrillation of the European Society of Cardiology (ESC) Developed with the special contribution of the European Heart Rhythm Association (EHRA) of the ESC. Eur Heart J 2021, 42(5): 373-498.

2. Van Gelder IC, Groenveld HF, Crijns HJ, et al. Lenient versus strict rate control in patients with atrial fibrillation. N Engl J Med 2010, 362(15): 1363-1373.

3. Corley SD, Epstein AE, DiMarco JP, et al. Relationships between sinus rhythm, treatment, and survival in the Atrial Fibrillation Follow-Up Investigation of Rhythm Management (AFFIRM) Study. Circulation 2004, 109(12): 1509-1513 
4. Groenveld HF, Crijns HJ, Van den Berg MP, et al. Investigators RI: Rate control efficacy in permanent atrial fibrillation: successful and failed strict rate control against a background of lenient rate control: data from RACE II (Rate Control Efficacy in Permanent Atrial Fibrillation). J Am Coll Cardiol 2013, 61(7): 741-748.

5. Groenveld HF, Tijssen JG, Crijns HJ, et al. Van Veldhuisen DJ, Van Gelder IC, Investigators RI: Rate control efficacy in permanent atrial fibrillation: successful and failed strict rate control against a background of lenient rate control: data from RACE II (Rate Control Efficacy in Permanent Atrial Fibrillation). J Am Coll Cardiol 2013, 61(7): 741-748.

6. Chen S, Purerfellner H, Meyer $\mathrm{C}$, et al. Rhythm control for patients with atrial fibrillation complicated with heart failure in the contemporary era of catheter ablation: a stratified pooled analysis of randomized data. Eur Heart J 2020, 41(30): 2863-2873.

7. Kornej J, Hindricks G, Shoemaker MB, et al. The APPLE score: a novel and simple score for the prediction of rhythm outcomes after catheter ablation of atrial fibrillation. Clin Res Cardiol 2015, 104(10): 871-876.

8. January CT, Wann LS, Calkins H, et al. 2019 AHA/ACC/HRS Focused Update of the 2014 AHA/ACC/HRS Guideline for the Management of Patients With Atrial Fibrillation: A Report of the American College of Cardiology/American Heart Association Task Force on Clinical Practice Guidelines and the Heart Rhythm Society in Collaboration With the Society of Thoracic Surgeons. Circulation 2019, 140(2): e125-e151.

9. Clark DM, Plumb VJ, Epstein AE, et al. Hemodynamic effects of an irregular sequence of ventricular cycle lengths during atrial fibrillation. J Am Coll Cardiol 1997, 30(4): 1039-1045

10. Tops LF, Schalij MJ, Bax JJ. The effects of right ventricular apical pacing on ventricular function and dyssynchrony implications for therapy. J Am Coll Cardiol 2009, 54(9): 764-776.

11. Wu S, Cai M, Zheng R, Wang S, et al. Impact of QRS morphology on response to conduction system pacing after atrioventricular junction ablation. ESC Heart Fail 2021, 8(2): 1195-1203.

12. Shan QJ, Xu H, Zhou XJ, et al. Effects of permanent left bundle branch area pacing on QRS duration and short-term cardiac function in pacing-indicated patients with left bundle branch block. Chin Med $\mathrm{J}$ (Engl) 2021, 134(9): 1101-1103.

13. Brignole $\mathrm{M}$, Pokushalov E, Pentimalli F, et al. A randomized controlled trial of atrioventricular junction ablation and cardiac resynchronization therapy in patients with permanent atrial fibrillation and narrow QRS. Eur Heart J 2018, 39(45): 3999-4008.

14. Michele Brignole, Francesco Pentimalli, Pietro Palmisano, et al. AV junction ablation and cardiac resynchronization for patients with permanent atrial fibrillation and narrow QRS: the APAF-CRT mortality trial. Eur Heart J 2021; doi: 10.1093/eurheartj/ehab569.

15. Young JB, Abraham WT, Smith AL, et al. Combined cardiac resynchronization and implantable cardioversion defibrillation in advanced chronic heart failure: the MIRACLE ICD Trial. JAMA 2003, 289(20): 2685-2694. 
16. Ghio S, Freemantle N, Serio A, et al. Baseline echocardiographic characteristics of heart failure patients enrolled in a large European multicentre trial (CArdiac REsynchronisation Heart Failure study). Eur J Echocardiogr 2006, 7(5): 373-378.

17. Zareba W, Klein H, Cygankiewicz I, et al. Effectiveness of Cardiac Resynchronization Therapy by QRS Morphology in the Multicenter Automatic Defibrillator Implantation Trial-Cardiac Resynchronization Therapy (MADIT-CRT). Circulation 2011, 123(10): 1061-1072.

\section{Tables}

\section{Table 1 Inclusion and exclusion criteria}

Inclusion criteria

1. Unsuitable or rejected for AF catheter ablation and failure in rhythm control

2. Persistent $A F$ (resting heart rate $\leq 110 \mathrm{bpm}$ ) combined with $\mathrm{HF}(\mathrm{LVEF}<50 \%)$ after optimal drug treatment for at least 3 months

3. Narrow QRS wave on ECG ( $\leq 120 \mathrm{~ms})$

4. Age $\bigotimes 18$ years and the expectancy life over 1 year

5. Agree to participate in the clinical trial and sign a written informed consent

Exclusion criteria

1. History of an acute coronary syndrome, cerebrovascular accident, and gastrointestinal bleeding within the last 3 months

2. Other serious heart diseases requiring surgical treatment

3. Any contraindications to ventricular rhythm resetting therapy

4. Pregnant or preparing to become pregnant or mentally ill

\section{Table 2 Six-item Specific Symptom Scale score}




\begin{tabular}{|ll|}
\hline & Score \\
\hline Palpitations & $0-10$ \\
\hline Effort dyspnoea & $0-10$ \\
\hline Rest dyspnoea & $0-10$ \\
\hline Effort intolerance & $0-10$ \\
\hline Easy fatigue & $0-10$ \\
\hline Chest discomfort & $0-10$ \\
\hline
\end{tabular}

Table 3 Advantages and disadvantages of different forms of APT

\begin{tabular}{|llllll|}
\hline & $\begin{array}{l}\text { Physiological } \\
\text { pacing mode }\end{array}$ & $\begin{array}{l}\text { Technical } \\
\text { difficulty }\end{array}$ & $\begin{array}{l}\text { Symptoms } \\
\text { improvement }\end{array}$ & $\begin{array}{l}\text { Rehospitalization } \\
\text { Rate }\end{array}$ & mortality \\
\hline AVNA+RVAP & $\mathrm{N}$ & + & $\uparrow$ & - & - \\
\hline AVNA+BIVP & Partly & +++ & $\uparrow$ & $\hat{a}$ & $\hat{a}$ \\
\hline AVNA+HBP & Y & ++ & $\uparrow$ & NA & NA \\
\hline AVNA+LBBP & Y & + & $\uparrow$ & NA & NA \\
\hline
\end{tabular}

APT: ablation and pacing therapy; AVNA: atrioventricular node ablation; RVAP: right ventricular apical pacing; BIVP: biventricular pacing; HBP: his bundle pacing; LBBP: left bundle branch pacing; N: not; Y: yes; +: relatively diffıcult; ++: diffıcult; +++: very diffıcult; $\uparrow$ : symptom improvement;â: positive outcome; --: neutral outcome; NA: not available

\section{Figures}




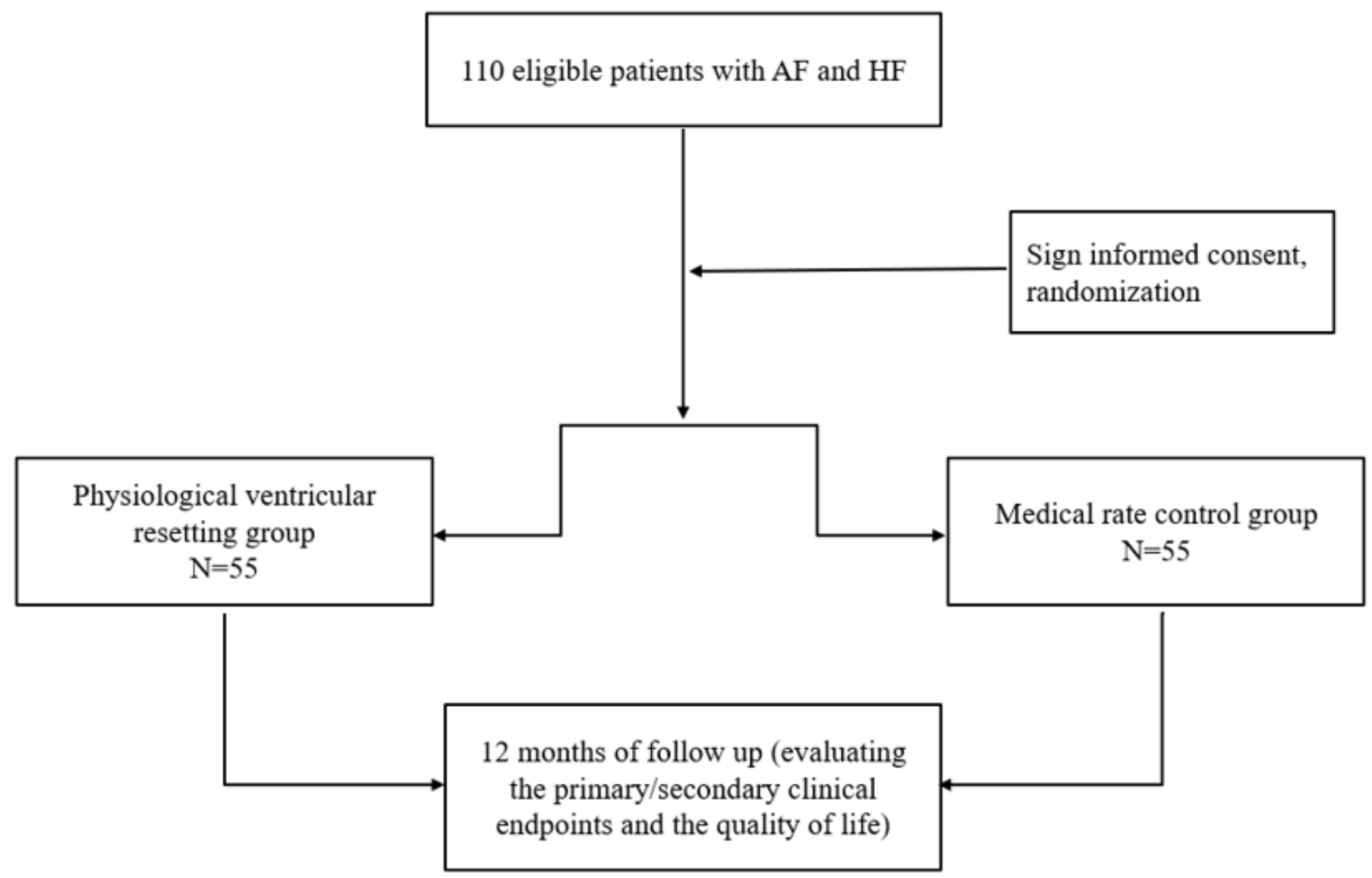

Figure 1

Flowchart of the physiological ventricular rhythm resetting trial 